\title{
Fixed points for $G$-contractions on uniform spaces endowed with a graph
}

\author{
Aris Aghanians, Kamal Fallahi and Kourosh Nourouzi*
}

${ }^{\text {*Correspondence: }}$

nourouzi@kntu.ac.ir

Department of Mathematics, K. N.

Toosi University of Technology,

P.O. Box 16315-1618, Tehran, Iran

\begin{abstract}
In this paper, we generalize some main results of (Jachymski in Proc. Am. Math. Soc. 136:1359-1373, 2008) from metric to uniform spaces endowed with an $\boldsymbol{E}$-distance and a graph using a new type of contractions by employing a class of nondecreasing functions.

MSC: $47 \mathrm{H} 10 ; 05 \mathrm{C} 40$

Keywords: weakly connected graph; $(p, \varphi)$-G-contraction; $p$-Picard operator; orbital $p$-continuity; graph orbital $p$-continuity
\end{abstract}

\section{Introduction and preliminaries}

In 2004, the concepts of $\mathcal{E}$-distance and $S$-completeness were introduced for uniform spaces in [1]. Recently in 2008, Jachymski [2] proved some fixed point results in metric spaces endowed with a graph and generalized simultaneously the Banach contraction principle from metric and partially ordered metric spaces. In 2010, Bojor [3] introduced $(G, \varphi)$-contractions and generalized Jachymski's results. Finally, Nicolae et al. [4] presented some fixed point results for a new type of contractions using orbits and also for $G$-asymptotic contractions in metric spaces endowed with a graph.

The aim of this paper is to generalize Theorems 3.1, 3.2, 3.3 and 3.4 and Proposition 3.1 of Jachymski [2] from metric to uniform spaces endowed with a graph and to present a new type of contractive mappings. The reader interested in (ordered) uniform fixed point theorems may consult the references [5-7].

Following Willard [8], a uniformity on a nonempty set $X$ is a nonempty family $U$ of subsets of $X \times X$ satisfying the following conditions:

(U1) Each $U \in \mathcal{U}$ contains the diagonal $\Delta(X)=\{(x, x): x \in X\}$;

(U2) $U$ is closed under finite intersections;

(U3) For each $U \in \mathcal{U}$, the set $\{(x, y):(y, x) \in U\}$ is a member of $\mathcal{U}$;

(U4) For each $U \in U$, there exists a member $V$ of $\mathcal{U}$ such that $(x, z) \in U$ whenever $(x, y),(y, z) \in V$ for all $x, y, z \in X$;

(U5) $U$ contains the supersets of its elements.

Given a uniformity $U$ on a nonempty set $X$, the pair $(X, \mathcal{U})$, simply denoted by $X$, is called a uniform space.

A uniformity $U$ on a nonempty set $X$ is separating if the intersection of all members of $\mathcal{U}$ is equal to $\Delta(X)$. In this case, $X$ is called a separated uniform space.

We are now ready to recall the concepts of $\mathcal{E}$-distance and $p$-completeness introduced by Aamri and El Moutawakil [1].

(c) 2012 Aghanians et al.; licensee Springer. This is an Open Access article distributed under the terms of the Creative Commons Attribution License (http://creativecommons.org/licenses/by/2.0), which permits unrestricted use, distribution, and reproduction in any medium, provided the original work is properly cited. 
Definition 1 ([1]) Let $X$ be a uniform space. A function $p: X \times X \rightarrow[0,+\infty)$ is said to be an $\mathcal{E}$-distance on $X$ if

(i) for each $U \in \mathcal{U}$, there exists a positive number $\delta$ such that $(x, y) \in U$ whenever $p(z, x) \leq \delta$ and $p(z, y) \leq \delta$ for all $x, y, z \in X$;

(ii) $p$ satisfies the triangular inequality, i.e.,

$$
p(x, y) \leq p(x, z)+p(z, y) \quad(x, y, z \in X) .
$$

A sequence $\left\{x_{n}\right\}$ in a uniform space $X$ equipped with an $\mathcal{E}$-distance $p$ is said to be $p$-convergent to a point $x \in X$, denoted by $x_{n} \stackrel{p}{\rightarrow} x$, if $p\left(x_{n}, x\right) \rightarrow 0$ as $n \rightarrow \infty$, and $p$-Cauchy if $p\left(x_{m}, x_{n}\right) \rightarrow 0$ as $m, n \rightarrow \infty$. The uniform space $X$ is said to be $p$-complete if each $p$-Cauchy sequence in $X$ is $p$-convergent to some point of $X$.

The next lemma shows that in a separated uniform space every sequence is $p$-convergent to at most one point. The proof is straightforward, and hence it is omitted here.

Lemma 1 ([1]) Let $\left\{x_{n}\right\}$ be a sequence in a separated uniform space $X$ equipped with an $\mathcal{E}$-distance $p$, and $x, y \in X$. If $x_{n} \stackrel{p}{\rightarrow} x$ and $x_{n} \stackrel{p}{\rightarrow} y$, then $x=y$. In particular, if $p(z, x)=$ $p(z, y)=0$ for some $z \in X$, then $x=y$.

Example 1 Suppose that the set $X=[0,+\infty)$ is endowed with the trivial uniformity, that is, $\mathcal{U}=\{X \times X\}$. Putting $p(x, y)=y$ for all $x, y \in X$, it is seen that $p$ is an $\mathcal{E}$-distance on $X$ and each sequence (and even each net) in $X$ is $p$-convergent only to zero, but clearly, this space is not separated. Therefore, the converse of Lemma 1 is not true in general.

We next review some basic notions of graph theory in relation to uniform spaces that we need in the sequel. For more details on the theory of graphs, see, e.g., [9].

Let $X$ be a uniform space equipped with an $\&$-distance $p$ and consider a directed graph $G$ with $V(G)=X$ and $\Delta(X) \subseteq E(G)$, that is, $E(G)$ contains all loops. Suppose further that $G$ has no parallel edges. The graph $G$ may be considered a weighted graph by assigning the nonnegative number $p(x, y)$ to each edge $(x, y)$ in $E(G)$.

We denote by $G^{-1}$ the conversion of the graph $G$, that is, $V\left(G^{-1}\right)=V(G)$ and

$$
E\left(G^{-1}\right)=\{(x, y) \in X \times X:(y, x) \in E(G)\} .
$$

The undirected graph obtained from $G$ by ignoring the directions of the edges of $G$ is denoted by $\widetilde{G}$. Indeed, $\widetilde{G}$ can be treated as a directed graph for which the set $E(\widetilde{G})$ is a symmetric subset of $X \times X$, namely $E(\widetilde{G})=E(G) \cup E\left(G^{-1}\right)$.

If $x$ and $y$ are two vertices in a graph $G$, then a path in $G$ from $x$ to $y$ is a finite sequence $\left(x_{i}\right)_{i=0}^{N}$ consisting of $N+1$ vertices of $G$ such that $x_{0}=x, x_{N}=y$, and $\left(x_{i-1}, x_{i}\right) \in E(G)$ for $i=1, \ldots, N$, where $N \in \mathbb{N}$. A graph $G$ is said to be connected if there exists a path in $G$ between each two vertices of $G$, and weakly connected if the graph $\widetilde{G}$ is connected.

By a subgraph of $G$, we mean a graph $H$ satisfying $V(H) \subseteq V(G)$ and $E(H) \subseteq E(G)$ such that $V(H)$ contains the vertices of all edges of $E(H)$.

If $x \in V(G)$ and $E(G)$ is symmetric, then the subgraph $G_{x}$ consisting of all edges and vertices that are contained in some path in $G$ that starts at $x$ is called the component of $G$ containing $x$. Thus, $V\left(G_{x}\right)=[x]_{G}$, where $[x]_{G}$ is the equivalence class of $x$ in the equivalence 
relation $\sim$ defined by

$$
y \sim z \quad \Longleftrightarrow \quad \text { there exists a path in } G \text { from } y \text { to } z \quad(y, z \in V(G)) .
$$

It is clear that the graph $G_{x}$ is connected for all $x \in X$.

\section{Main results}

Throughout this section, we assume that $X$ is a uniform space that is endowed with an $\mathcal{E}$-distance $p$ and a directed graph $G$ with $V(G)=X$ and $\Delta(X) \subseteq E(G)$ unless stated otherwise.

We denote by $\operatorname{Fix}(f)$ the set of all fixed points for a self-map $f$ on $X$, and further by $\Phi$ the class of all nondecreasing functions $\varphi$ from $[0,+\infty)$ into $[0,1)$.

Following Jachymski [2], we introduce $(p, \varphi)$-G-contractions on a uniform space endowed with an $\mathscr{E}$-distance and a graph.

Definition 2 Let $f$ be a self-map on $X$ and $\varphi$ be a function in $\Phi$. Then $f$ is called a $(p, \varphi)$ $G$-contraction if

(C1) the edges of $G$ are preserved by $f$, i.e., $(x, y) \in E(G)$ implies $(f x, f y) \in E(G)$ for all $x, y \in X$

(C2) the $p$-weights of the edges of $G$ are $\varphi$-decreased by $f$ in the sense that

$$
p(f x, f y) \leq \varphi(p(x, y)) p(x, y)
$$

for all $x, y \in X$ with $(x, y) \in E(G)$.

If $p$ is a metric on $X$, then we call $f$ a $\varphi$-G-contraction, and we call $f$ a $(p, \varphi)$-contraction if (1) holds for all $x, y \in X$.

We now give some examples of $(p, \varphi)$-G-contractions.

Example 2 If $p\left(x_{0}, x_{0}\right)=0$ for some $x_{0} \in X$, since $E(G)$ contains all loops, it follows that the constant mapping $f=x_{0}$ is a $(p, \varphi)$-G-contraction for any $\varphi \in \Phi$. In particular, $p(x, x)=0$ for all $x \in X$ if and only if each constant mapping on $X$ is a $(p, \varphi)$-G-contraction for some $\varphi \in \Phi$.

Example 3 Each $(p, \varphi)$-contraction is a $(p, \varphi)$ - $G_{0}$-contraction, where $G_{0}$ is the complete graph with $V\left(G_{0}\right)=X$, that is, $E\left(G_{0}\right)=X \times X$.

Example 4 Suppose that $\preceq$ is a partial order on $X$ and consider the poset graph $G_{1}$, that is, $V\left(G_{1}\right)=X$ and

$$
E\left(G_{1}\right)=\{(x, y) \in X \times X: x \preceq y\} .
$$

Then Condition (C1) means that $f$ is nondecreasing with respect to $\preceq$, and Condition (C2) means that $f$ is an order $(p, \varphi)$-contraction, i.e., (1) holds for all $x, y \in X$ with $x \preceq y$.

In the next example, we construct a self-map $f$ that fails to be a $\varphi$-G-contraction for any $\varphi \in \Phi$, whereas $f$ is a $(p, \varphi)$-G-contraction for some $\mathcal{E}$-distance $p$ and some $\varphi \in \Phi$. 
Example 5 Let $X=[0,+\infty)$ be endowed with the usual uniformity (see [8, p.239]) and the graph $G_{0}$. Let $n \geq 2$ and consider a self-map $f$ on $X$ defined by the rule

$$
f x= \begin{cases}0, & 0 \leq x<1 \\ \frac{\sqrt[n]{x}+1}{3}, & x \geq 1\end{cases}
$$

Then $f$ is not a $\varphi-G_{0}$-contraction for any $\varphi \in \Phi$. Because, setting $x=1$ and $y=\frac{1}{2}$, we have

$$
\left|f 1-f \frac{1}{2}\right|=\frac{2}{3}>\frac{1}{2} \times \varphi\left(\frac{1}{2}\right)=\varphi\left(\left|1-\frac{1}{2}\right|\right)\left|1-\frac{1}{2}\right| .
$$

Now, put $p(x, y)=y$ for all $x, y \in X$ and define $\varphi:[0,+\infty) \rightarrow[0,1)$ by

$$
\varphi(t)= \begin{cases}\frac{t^{2}}{3}, & 0 \leq t<1, \\ \frac{2}{3}, & t \geq 1 .\end{cases}
$$

Clearly, $\varphi \in \Phi$ and an easy argument shows that Conditions (C1) and (C2) are satisfied. Thus, $f$ is a $(p, \varphi)-G_{0}$-contraction.

Example 6 Consider the mapping $f$ on $\mathbb{R}$ defined by $f x=x+1$ for all $x \in \mathbb{R}$. Let $G$ be an arbitrary weakly connected graph with $V(G)=\mathbb{R}$ and $\Delta(\mathbb{R}) \subseteq E(G)$ and $\varphi$ be any function in $\Phi$. Since $G$ is weakly connected, there exists two distinct points $x, y \in \mathbb{R}$ such that $(x, y) \in$ $E(G)$. Then

$$
|f x-f y|=|x-y|>\varphi(|x-y|)|x-y| .
$$

Therefore, $f$ is not a $\varphi$-G-contraction for any weakly connected graph $G$ (with $V(G)=$ $\mathbb{R}$ and $\Delta(\mathbb{R}) \subseteq E(G))$ and any function $\varphi \in \Phi$. Now let $\mathbb{R}$ be equipped with the trivial uniformity $U=\{X \times X\}$. Then $p: \mathbb{R} \times \mathbb{R} \rightarrow[0,+\infty)$ defined by $p(x, y)=0$ for all $x, y \in \mathbb{R}$ is an $E$-distance on $\mathbb{R}$, and the mapping $f$ is a $(p, \varphi)$ - $\widetilde{G_{0}}$-contraction for any function $\varphi \in \Phi$.

Example 7 Let $X=[0,1]$ be endowed with the usual uniformity and define $f: X \rightarrow X$ by

$$
f x= \begin{cases}\frac{1}{4}, & 0 \leq x<1, \\ \frac{1}{8}, & x=1 .\end{cases}
$$

Then $f$ is not a $\varphi-G_{0}$-contraction for any $\varphi \in \Phi$ since

$$
|f x-f 1|=\frac{1}{8}>\varphi(1-x)(1-x)=\varphi(|x-1|)|x-1| \quad\left(\frac{7}{8}<x<1\right) .
$$

On the other hand, defining ' $\preceq$ ' by

$$
x \leq y \quad \Longleftrightarrow \quad\left((x=y) \vee\left(x, y \in\left[\frac{7}{8}, 1\right], x \leq y\right)\right) \quad(x, y \in X),
$$

the same argument shows that $f$ fails to be a $\varphi-G_{1}$-contraction for any $\varphi \in \Phi$. 
Now, set $V\left(G_{3}\right)=X$ and

$$
E\left(G_{3}\right)=\Delta(X) \cup\left\{\left(\frac{1}{n}, \frac{1}{n+1}\right): n \geq 1\right\} \cup\left\{\left(\frac{1}{4}, \frac{1}{8}\right),\left(\frac{1}{8}, \frac{1}{4}\right)\right\} .
$$

Then $f$ is a $\varphi-G_{3}$-contraction if we define $\varphi:[0,+\infty) \rightarrow[0,1)$ by the rule $\varphi(t)=\frac{4 t}{7}$.

Remark 1 It is worth mentioning that Conditions $(\mathrm{C} 1)$ and $(\mathrm{C} 2)$ are independent of each other. For instance, the identity mapping on $\mathbb{R}$ preserves the edges of $G_{0}$, but there is no $\varphi \in \Phi$ for which the contractive condition (1) holds. Conversely, setting $f x=-\frac{1}{2} x$ for all $x \in \mathbb{R}$, it is seen that $f$ is an order $(p, \varphi)$-contraction for the constant function $\varphi=\frac{1}{2}$ but $f$ fails to be nondecreasing.

Remark 2 Putting $\varphi$ the constant function $\alpha \in[0,1)$ in Definition 2, we get the concept of Banach $G$-contraction with $p=d$ on a metric space $(X, d)$, which was introduced by Jachymski [2].

Definition 3 We say that the sequences $\left\{x_{n}\right\}$ and $\left\{y_{n}\right\}$ are $p$-Cauchy equivalent in $X$ if both of them are $p$-Cauchy and further, $p\left(x_{n}, y_{n}\right) \rightarrow 0$ as $n \rightarrow \infty$.

Hereafter we assume that $\varphi$ is an arbitrary fixed function in $\Phi$ and $f: X \rightarrow X$ is a $(p, \varphi)$ $\widetilde{G}$-contraction unless stated otherwise.

Theorem 1 Consider the following statements:

(A) $G$ is weakly connected.

(B) Iff is a $(p, \varphi)-\widetilde{G}$-contraction, then $\left\{f^{n} x\right\}$ and $\left\{f^{n} y\right\}$ are $p$-Cauchy equivalent for all $x, y \in X$.

(C) Each $(p, \varphi)-\widetilde{G}$-contraction has at most one fixed point in $X$.

Then,

(i) (A) implies (B).

(ii) (B) implies (C) provided that $X$ is separated.

(iii) (C) implies (A) provided that $p(x, x)=0$ for all $x \in X$.

Proof (i) Let $x, y \in X$ be given. Since $G$ is weakly connected, there exists a path $\left(x_{i}\right)_{i=0}^{N}$ in $\widetilde{G}$ from $x$ to $y$, i.e., $x_{0}=x, x_{N}=y$, and $\left(x_{i-1}, x_{i}\right) \in E(\widetilde{G})$ for each $i$. Because $f$ is a $(p, \varphi)$ $\widetilde{G}$-contraction, we get by induction that

$$
\left(f^{n} x_{i-1}, f^{n} x_{i}\right) \in E(\widetilde{G}) \quad(n \geq 1)
$$

and, furthermore, by the contractive condition (1) and the fact that $\varphi$ is nondecreasing, we get

$$
\begin{aligned}
p\left(f^{n} x_{i-1}, f^{n} x_{i}\right) & \leq \varphi\left(p\left(f^{n-1} x_{i-1}, f^{n-1} x_{i}\right)\right) p\left(f^{n-1} x_{i-1}, f^{n-1} x_{i}\right) \\
& \leq \varphi\left(p\left(f^{n-1} x_{i-1}, f^{n-1} x_{i}\right)\right) \varphi\left(p\left(f^{n-2} x_{i-1}, f^{n-2} x_{i}\right)\right) p\left(f^{n-2} x_{i-1}, f^{n-2} x_{i}\right)
\end{aligned}
$$




$$
\begin{aligned}
& \leq \prod_{k=1}^{n} \varphi\left(p\left(f^{n-k} x_{i-1}, f^{n-k} x_{i}\right)\right) p\left(x_{i-1}, x_{i}\right) \\
& \leq\left(\varphi\left(p\left(x_{i-1}, x_{i}\right)\right)\right)^{n} p\left(x_{i-1}, x_{i}\right)
\end{aligned}
$$

for all $n \geq 1$ and all $i=1, \ldots, N$. Hence, by the triangle inequality of $p$,

$$
\begin{aligned}
p\left(f^{n} x, f^{n} y\right) & \leq \sum_{i=1}^{N} p\left(f^{n} x_{i-1}, f^{n} x_{i}\right) \\
& \leq \sum_{i=1}^{N}\left(\varphi\left(p\left(x_{i-1}, x_{i}\right)\right)\right)^{n} p\left(x_{i-1}, x_{i}\right) \\
& \leq k^{n} r(x, y) \rightarrow 0 \quad \text { as } n \rightarrow \infty,
\end{aligned}
$$

where $k=\max \left\{\varphi\left(p\left(x_{i-1}, x_{i}\right)\right): i=1, \ldots, N\right\}<1$ and $r(x, y)=\sum_{i=1}^{N} p\left(x_{i-1}, x_{i}\right)$. Now, the weak connectivity of $G$ gives $f x \in X=[x]_{\widetilde{G}}$, and so, setting $y=f x$ in (2) yields

$$
p\left(f^{n} x, f^{n+1} x\right) \leq k^{n} r(x, f x)
$$

for all $n \geq 1$. Since $k<1$, it follows that $\sum_{n=0}^{\infty} p\left(f^{n} x, f^{n+1} x\right)<\infty$ and a standard argument shows that $\left\{f^{n} x\right\}$ is $p$-Cauchy. Similarly, $\left\{f^{n} y\right\}$ is $p$-Cauchy and hence $\left\{f^{n} x\right\}$ and $\left\{f^{n} y\right\}$ are $p$-Cauchy equivalent.

(ii) Let $x$ and $y$ be two fixed points for $f$. Since, by the hypothesis, $\left\{f^{n} x\right\}$ and $\left\{f^{n} y\right\}$ are $p$-Cauchy equivalent, it follows that

$$
p(x, y)=p\left(f^{n} x, f^{n} y\right) \rightarrow 0 \quad \text { and } \quad p(x, x)=p\left(f^{n} x, f^{n} x\right) \rightarrow 0
$$

Because $X$ is separated, Lemma 1 ensures that $x=y$.

(iii) Suppose on the contrary that $G$ is not weakly connected. Then there exists an $x_{0} \in X$ such that both sets $\left[x_{0}\right]_{\widetilde{G}}$ and $X \backslash\left[x_{0}\right]_{\widetilde{G}}$ are nonempty. Fix any $y_{0} \in X \backslash\left[x_{0}\right]_{\widetilde{G}}$ and define $f: X \rightarrow X$ by

$$
f x= \begin{cases}x_{0}, & x \in\left[x_{0}\right] \widetilde{G}, \\ y_{0}, & x \in X \backslash\left[x_{0}\right] \widetilde{G}\end{cases}
$$

Clearly, $\operatorname{Fix}(f)=\left\{x_{0}, y_{0}\right\}$. To get a contradiction, we show that $f$ is a $(p, \varphi)-\widetilde{G}$-contraction. If $(x, y) \in E(\widetilde{G})$, then $[x]_{\widetilde{G}}=[y]_{\widetilde{G}}$, and so either $x, y \in\left[x_{0}\right]_{\widetilde{G}}$ or $x, y \in X \backslash\left[x_{0}\right]_{\widetilde{G}}$. Hence, in both cases, $f x=f y$, so $(f x, f y) \in E(\widetilde{G})$ because $\Delta(X) \subseteq E(\widetilde{G})$. Moreover,

$$
p(f x, f y)=0 \leq \varphi(p(x, y)) p(x, y)
$$

where $\varphi$ is any arbitrary function in $\Phi$. Thus, $f$ is a $(p, \varphi)-\widetilde{G}$-contraction.

Corollary 1 If $X$ is p-complete and $G$ is weakly connected, then there exists an $x^{*} \in X$ such that $f^{n} \stackrel{p}{\rightarrow} x^{*}$ for all $x \in X$. 
Proof Fix any point $x \in X$. By Theorem $1,\left\{f^{n} x\right\}$ is a $p$-Cauchy sequence in $X$. Since $X$ is $p$-complete, there exists an $x^{\prime \prime} \in X$ such that $f^{n} x \stackrel{p}{\rightarrow} x^{*}$. Now, if $y \in X$, then by the $p$-Cauchy equivalence of $\left\{f^{n} y\right\}$ and $\left\{f^{n} x\right\}$, we have

$$
p\left(f^{n} y, x^{*}\right) \leq p\left(f^{n} y, f^{n} x\right)+p\left(f^{n} x, x^{*}\right) \rightarrow 0 .
$$

Therefore, $f^{n} y \stackrel{p}{\rightarrow} x^{*}$.

Proposition 1 If for some $x_{0} \in X$ we have $f x_{0} \in\left[x_{0}\right]_{\widetilde{G}}$, then $\left[x_{0}\right]_{\widetilde{G}}$ is $f$-invariant and $\left.f\right|_{\left[x_{0}\right]_{\tilde{G}}}$ is a $(p, \varphi)-\widetilde{G}_{x_{0}}$-contraction. Moreover, if $x, y \in\left[x_{0}\right]_{\widetilde{G}}$, then $\left\{f^{n} x\right\}$ and $\left\{f^{n} y\right\}$ are $p$-Cauchy equivalent.

Proof Let $x \in\left[x_{0}\right]_{\widetilde{G}}$. Then there exists a path $\left(x_{i}\right)_{i=0}^{N}$ in $\widetilde{G}$ from $x_{0}$ to $x$, i.e., $x_{N}=x$ and $\left(x_{i-1}, x_{i}\right) \in E(\widetilde{G})$ for each $i$. So, $\left(f x_{i-1}, f x_{i}\right) \in E(\widetilde{G})$ for each $i$, that is, $\left(f x_{i}\right)_{i=0}^{N}$ is a path in $\widetilde{G}$ from $f x_{0}$ to $f x$, and since $f x_{0} \in\left[x_{0}\right]_{\widetilde{G}}$, there is another path $\left(y_{j}\right)_{j=0}^{M}$ in $\widetilde{G}$ from $x_{0}$ to $f x_{0}$, i.e., $y_{0}=x_{0}$, $y_{M}=f x_{0}$, and $\left(y_{j-1}, y_{j}\right) \in E(\widetilde{G})$ for each $j$. Thus, $f x \in\left[x_{0}\right]_{\widetilde{G}}$. Therefore, $\left[x_{0}\right]_{\widetilde{G}}$ is $f$-invariant.

Next, let $(x, y) \in E\left(\widetilde{G}_{x_{0}}\right)$. This means that there exists a path $\left(x_{i}\right)_{i=0}^{N}$ in $\widetilde{G}$ from $x_{0}$ to $y$ such that $x_{N-1}=x$. Repeating the argument above, we infer that

$$
\left(x_{0}=y_{0}, y_{1}, \ldots, f x_{0}=y_{M}, f x_{1}, \ldots, f y=f x_{N}\right)
$$

is a path in $\widetilde{G}$ from $x_{0}$ to $f y$. In particular,

$$
(f x, f y)=\left(f x_{N-1}, f x_{N}\right) \in E\left(\widetilde{G}_{x_{0}}\right) .
$$

Since $E\left(\widetilde{G}_{x_{0}}\right) \subseteq E(\widetilde{G})$, it follows that $f$ is a $(p, \varphi)-\widetilde{G}_{x_{0}}$-contraction.

Moreover, because $\widetilde{G}_{x_{0}}$ is weakly connected, Theorem 1 implies that the sequences $\left\{f^{n} x\right\}$ and $\left\{f^{n} y\right\}$ are $p$-Cauchy equivalent for all $x, y \in\left[x_{0}\right]_{\tilde{G}}$.

Following Petruşel and Rus [10], we introduce the concept of a $p$-Picard operator.

Definition 4 A self-map $f$ on $X$ is called a $p$-Picard operator if $f$ has a unique fixed point $x^{*}$ in $X$ and $f^{n} x \stackrel{p}{\rightarrow} x^{*}$ for all $x \in X$.

Given a self-map $f$ on $X$, we denote the set $\{x \in X:(x, f x) \in E(\widetilde{G})\}$ by $X_{f}$.

Theorem 2 Let X be p-complete, separated and satisfy the following property:

(*) For each sequence $\left\{x_{n}\right\}$ in $X$, $p$-convergent to some $x \in X$, if $\left(x_{n}, x_{n+1}\right) \in E(\widetilde{G})$ for all $n \geq 1$, then there exists a subsequence $\left\{x_{n_{k}}\right\}$ of $\left\{x_{n}\right\}$ such that $\left(x_{n_{k}}, x\right) \in E(\widetilde{G})$ for all $k \geq 1$.

Then

(i) $\left.f\right|_{[x]_{\tilde{G}}}$ is a p-Picard operator for each $x \in X_{f}$.

(ii) If $X_{f} \neq \emptyset$ and $G$ is weakly connected, $f$ is a $p$-Picard operator.

(iii) $\operatorname{card}(\operatorname{Fix}(f))=\operatorname{card}\left\{[x]_{\tilde{G}}: x \in X_{f}\right\}$.

(iv) $\operatorname{Fix}(f) \neq \emptyset$ if and only if $X_{f} \neq \emptyset$.

(v) $f$ has a unique fixed point if and only if there exists an $x_{0} \in X_{f}$ such that $X_{f} \subseteq\left[x_{0}\right]_{\widetilde{G}}$. 
Proof (i) Let $x \in X_{f}$. Then $f x \in[x]_{\widetilde{G}}$, and so by Proposition 1 and Corollary 1 , there exists an $x^{*} \in X$ such that $f^{n} y \stackrel{p}{\rightarrow} x^{*}$ for all $y \in[x]_{\widetilde{G}}$. Since $(x, f x) \in E(\widetilde{G})$, it follows from Condition (C1) that

$$
\left(f^{n} x, f^{n+1} x\right) \in E(\widetilde{G}) \quad(n \geq 1) .
$$

By the property $(*)$, there exists a subsequence $\left\{f^{n_{k}} x\right\}$ of $\left\{f^{n} x\right\}$ such that $\left(f^{n_{k}} x, x^{*}\right) \in E(\widetilde{G})$ for all $k \geq 1$.

Now, by (3), we see that $\left(x, f x, \ldots, f^{n_{1}} x, x^{*}\right)$ is a path in $\widetilde{G}$ from $x$ to $x^{\prime \prime}$, that is, $x^{\prime \prime} \in[x]_{\widetilde{G}}$. Moreover, because $f$ is a $(p, \varphi)-\widetilde{G}$-contraction, it follows that

$$
p\left(f^{n_{k}+1} x, f x^{*}\right) \leq \varphi\left(p\left(f^{n_{k}} x, x^{*}\right)\right) p\left(f^{n_{k}} x, x^{\prime \prime}\right) \quad(k \geq 1) .
$$

Letting $k \rightarrow \infty$, it is concluded that $f^{n_{k}+1} x \stackrel{p}{\rightarrow} f x^{\prime \prime}$, and since $f^{n_{k}+1} x \stackrel{p}{\rightarrow} x^{*}$, Lemma 1 yields $x^{\prime \prime}=f x^{*}$. If $y^{\prime \prime} \in[x]_{\widetilde{G}}$ is a fixed point for $f$, since $f^{n} y^{\prime \prime} \stackrel{p}{\rightarrow} x^{\prime \prime}$, it follows that $p\left(y^{\prime \prime}, x^{\prime \prime}\right)=0$. Furthermore, since, by Proposition $1,\left\{f^{n} y^{\prime \prime}\right\}$ is $p$-Cauchy equivalent to itself, it follows that $p\left(y^{*}, y^{*}\right)=0$. Now, again by Lemma $1, x^{*}=y^{*}$. Thus, $\left.f\right|_{[x] \widetilde{G}}$ is a $p$-Picard operator.

(ii) If $G$ is weakly connected, then $[x]_{\widetilde{G}}=X$, where $x \in X_{f}$, and so, by (i), $f$ is a $p$-Picard operator.

(iii) Set $C=\left\{[x]_{\widetilde{G}}: x \in X_{f}\right\}$ and define a mapping $\theta: \operatorname{Fix}(f) \rightarrow C$ by

$$
\theta(x)=[x]_{\widetilde{G}} .
$$

We are going to show that $\theta$ is a bijection. Since $\Delta(X) \subseteq E(\widetilde{G})$, it follows that $\operatorname{Fix}(f) \subseteq X_{f}$, and hence $\theta$ is well-defined. On the other hand, if $x \in X_{f}$, then by Theorem 1 , there exists an $x^{*} \in X$ such that

$$
f^{n} x \stackrel{p}{\rightarrow} x^{*} \in[x]_{\widetilde{G}} \cap \operatorname{Fix}(f),
$$

which implies that

$$
\theta\left(x^{*}\right)=\left[x^{*}\right]_{\widetilde{G}}=[x]_{\widetilde{G}} .
$$

Thus, $\theta$ is surjective.

Now, if $x_{1}, x_{2} \in \operatorname{Fix}(f)$ are such that $\theta\left(x_{1}\right)=\theta\left(x_{2}\right)$, then $x_{2} \in\left[x_{1}\right]_{\widetilde{G}}$. So, by Proposition 1 and Theorem 1 ,

$$
f^{n} x_{1}, f^{n} x_{2} \stackrel{p}{\rightarrow} x^{*} \in\left[x_{1}\right]_{\widetilde{G}} \cap \operatorname{Fix}(f)=\left\{x_{1}\right\}
$$

Therefore, $x_{1}=x^{\prime \prime}=x_{2}$. Consequently, $\theta$ is injective.

(iv), (v) They are immediate consequences of (iii).

Remark 3 In [11], Gwóźdź-Łukawska and Jachymski proved some fixed point results for iterated function systems of $G$-contractions, and in [11, Example 2], they explicitly claimed that none of [11, Theorems 4-7] can be generalized to the family of all edge-preserving self-maps satisfying the contractive condition

$$
d(f x, f y) \leq \varphi(d(x, y)) \quad((x, y) \in E(G)),
$$


where $\varphi: \mathbb{R}^{+} \rightarrow \mathbb{R}^{+}$is nondecreasing and satisfies $\varphi^{n}(t) \rightarrow 0$ for all $t>0$. More precisely, their example contains a fixed point free $(G, \varphi)$-contraction (in the sense of [3, Definition 2.1]) and hence it contradicts [3, Theorem 2.3, (2)], which implies the existence of a fixed point for such a mapping. It might be interesting for the reader why the mapping $f$ in this example does not satisfy (1), which is the main condition of Theorem 2 of this paper and so the example is not in contrary with that theorem. We give the following two reasons:

(1) Let $f$ and $\varphi$ be as in [11, Example 2]. Since for each $n \geq 1$, we have

$$
\left|f s_{n}-f s_{n+1}\right|=\varphi\left(\left|s_{n}-s_{n+1}\right|\right)>\varphi\left(\left|s_{n}-s_{n+1}\right|\right) \cdot \frac{1}{n+1}=\varphi\left(\left|s_{n}-s_{n+1}\right|\right) \cdot\left|s_{n}-s_{n+1}\right|
$$

it shows that $f$ is not a $\varphi$-G-contraction, i.e., it does not satisfy

$$
|f x-f y| \leq \varphi(|x-y|) \cdot|x-y| \quad((x, y) \in E(G)) .
$$

In particular, $f$ is not a $\varphi-\widetilde{G}$-contraction;

(2) In essence, the mapping $f$ in [11, Example 2] fails to be a $\varphi$-G-contraction for any $\varphi \in \Phi$. Otherwise, if $f$ is a $\varphi-G$-contraction for some $\varphi \in \Phi$, then from

$$
\frac{1}{n+2}=\left|f s_{n}-f s_{n+1}\right| \leq \varphi\left(\left|s_{n}-s_{n+1}\right|\right) \cdot\left|s_{n}-s_{n+1}\right|=\varphi\left(\frac{1}{n+1}\right) \cdot \frac{1}{n+1}
$$

we have

$$
\frac{n+1}{n+2} \leq \varphi\left(\frac{1}{n+1}\right), \quad n=1,2 \ldots
$$

By the monotonicity of $\varphi$, it follows that the sequence $\left\{\varphi\left(\frac{1}{n+1}\right)\right\}$ is nonincreasing and bounded from below. Therefore,

$$
1=\lim _{n \rightarrow \infty} \frac{n+1}{n+2} \leq \lim _{n \rightarrow \infty} \varphi\left(\frac{1}{n+1}\right) \leq \varphi\left(\frac{1}{2}\right)<1
$$

which is a contradiction.

Example 8 Let $X=\left\{\frac{1}{n}: n \geq 1\right\} \cup\left\{0, \frac{2}{3}\right\}$ be endowed with the Euclidean metric and define a graph $G_{4}$ by $V\left(G_{4}\right)=X$ and

$$
E\left(G_{4}\right)=\Delta(X) \cup\left\{\left(\frac{1}{n}, \frac{1}{n+1}\right): n \geq 1\right\} \cup\left\{\left(\frac{1}{n}, 0\right): n \geq 1\right\} \cup\left\{\left(\frac{2}{3}, 0\right),\left(0, \frac{2}{3}\right)\right\} .
$$

Clearly, $X$ is complete and $G_{4}$ is weakly connected. Consider $f$ defined by $f x=\frac{2}{3}$ if $x=1$, and $f x=0$ if $x \neq 1$. Then $f$ is edge-preserving but given $\alpha \in[0,1)$, it is seen that

$$
\left|f 1-f \frac{1}{2}\right|=\frac{2}{3}>\frac{\alpha}{2}=\alpha\left|1-\frac{1}{2}\right|
$$

So, $f$ is not a Banach $G_{4}$-contraction in the sense of [2, Definition 2.1] and we cannot use [2, Theorem 3.2]. 
Now, uniformize $X$ with the usual uniformity and consider an $\mathcal{E}$-distance $p$ on $X$ by $p(x, y)=y$. Then $X$ is separated, $p$-complete and satisfies the property $(*)$. Moreover, $X_{f}=X$. Therefore, considering $\varphi$ as in Example 5, it is seen that $f$ is a $(p, \varphi)-G_{4}$-contraction, and so by Theorem $2, f$ is a $p$-Picard operator with the unique fixed point zero.

Our next result is a generalization of [2, Corollary 3.2].

Corollary 2 Let $X$ be p-complete, separated and satisfy the property $(*)$, and $p(x, x)=0$ for all $x \in X$. Then the following statements are equivalent:

(i) $G$ is weakly connected;

(ii) Iff is a $(p, \varphi)$ - $\widetilde{G}$-contraction such that $\left(x_{0}, f x_{0}\right) \in E(\widetilde{G})$ for some $x_{0} \in X$, then $f$ is a p-Picard operator;

(iii) Each $(p, \varphi)$ - $\widetilde{G}$-contraction has at most one fixed point in $X$.

Proof (i) $\Rightarrow$ (ii): It follows immediately from Theorem 2 .

(ii) $\Rightarrow$ (iii): Let $f$ be a $(p, \varphi)-\widetilde{G}$-contraction. If $\operatorname{Fix}(f)=\emptyset$, then there is nothing to prove. Otherwise, by Theorem $2, X_{f} \neq \emptyset$. Thus, by the hypothesis, $f$ is a $p$-Picard operator and so it has a unique fixed point.

(iii) $\Rightarrow$ (i): It follows from Theorem 1 .

Following the idea of Jachymski [2], we define two different types of $p$-continuity of selfmaps on $X$ and then we discuss them.

Definition 5 Let $f$ be a self-map on $X$. We say that

(i) $f$ is orbitally $p$-continuous if for each $x, y \in X$ and each sequence $\left\{a_{n}\right\}$ of positive integers, $f^{a_{n}} x \stackrel{p}{\rightarrow} y$ implies $f\left(f^{a_{n}} x\right) \stackrel{p}{\rightarrow} f y$.

(ii) $f$ is graph orbitally $p$-continuous if for each $x, y \in X$ and each sequence $\left\{a_{n}\right\}$ of positive integers with $\left(f^{a_{n}} x, f^{a_{n+1}} x\right) \in E(\widetilde{G})$ for $n=1,2, \ldots$ such that $f^{a_{n}} x \stackrel{p}{\rightarrow} y$, one has $f\left(f^{a_{n}} x\right) \stackrel{p}{\rightarrow} f y$.

It is clear that $p$-continuity (see [1, Definition 2.3]) implies orbital $p$-continuity, and orbital $p$-continuity implies graph orbital $p$-continuity. But the converse of these relations is not true in general as the next example shows.

Example 9 Let $X=[0,1]$ be endowed with the uniformity induced by the usual metric.

(i) Consider an $\mathcal{E}$-distance $p$ on $X$ defined by $p(x, y)=\max \{x, y\}$ and a self-map $f$ defined by the rule $f x=1$ if $x \neq 0$ and $f 0=0$. Then $f$ is orbitally $p$-continuous since $\max \left\{f^{a_{n}} x, y\right\} \rightarrow 0$ implies that $x=y=0$. But $f$ fails to be $p$-continuous because $\frac{1}{n} \stackrel{p}{\rightarrow} 0$, whereas $f \frac{1}{n} \stackrel{p}{\rightarrow} f 0$.

(ii) Next, consider the $\mathcal{E}$-distance $p(x, y)=|x-y|$ on $X$ and the graph $G$ with $E(G)=\Delta(X)$. Then the self-map $f$ on $X$ defined by the rule $f x=\frac{x^{2}}{2}$ if $x \neq 0$ and $f 0=1$ is graph orbitally $p$-continuous since $\left(f^{a_{n}} x, f^{a_{n+1}} x\right) \in E(G)$ for all $n$ implies that $\left\{f^{a_{n}} x\right\}$ is a constant sequence. But setting $x=y=0$ and $a_{n}=n$, it is seen that $f^{a_{n}} x \stackrel{p}{\rightarrow} y$, whereas $f\left(f^{a_{n}} x\right) \stackrel{p}{\rightarrow} f y$.

Theorem 3 Let $X$ be p-complete and separated, and $f: X \rightarrow X$ be an orbitally $p$ continuous $(p, \varphi)-\widetilde{G}$-contraction. Then 
(i) for each $x \in X$ with $f x \in[x]_{\widetilde{G}}$, there exists an $x^{*} \in \operatorname{Fix}(f)$ such that $f^{n} y \stackrel{p}{\rightarrow} x^{*}$ for all $y \in[x]_{\widetilde{G}}$.

(ii) $\operatorname{Fix}(f) \neq \emptyset$ if and only if there exists an $x_{0} \in X$ such that $f x_{0} \in\left[x_{0}\right]_{\widetilde{G}}$.

(iii) If $G$ is weakly connected, $f$ is a $p$-Picard operator.

Proof (i) Let $x \in X$ be such that $f x \in[x]_{\widetilde{G}}$, and let $y \in[x]_{\tilde{G}}$. Since $X$ is $p$-complete, by Proposition $1,\left\{f^{n} x\right\}$ and $\left\{f^{n} y\right\}$ are $p$-convergent to a same point $x^{*} \in X$. Since $f$ is orbitally $p$-continuous, it follows that

$$
f\left(f^{n} x\right) \stackrel{p}{\rightarrow} f x^{*}
$$

On the other hand, $f\left(f^{n} x\right)=f^{n+1} x \stackrel{p}{\rightarrow} x^{*}$, and so by Lemma 1 , we have $x^{\prime \prime}=f x^{*}$, that is, $x^{*} \in \operatorname{Fix}(f)$. Moreover, if $z$ is any other point in $[x]_{\widetilde{G}}$, then it is clear that $f^{n} z \stackrel{p}{\rightarrow} x^{*}$.

(ii) If there exists an $x_{0} \in X$ such that $f x_{0} \in\left[x_{0}\right] \widetilde{G}$, then, by (i), we have Fix $(f) \neq \emptyset$. The converse is trivial.

(iii) Since $G$ is weakly connected, it follows that $[x]_{\widetilde{G}}=X$ for all $x \in X$. So, by (i), there exists an $x^{*} \in \operatorname{Fix}(f)$ such that $f^{n} y \stackrel{p}{\rightarrow} x^{*}$ for all $y \in X$. Now, similar to the proof of Theorem 2, one can show that $x^{*}$ is the only fixed point for $f$, and hence $f$ is a $p$-Picard operator.

A generalization of [2, Corollary 3.3] is given in the next result.

Corollary 3 Let $X$ be $p$-complete and separated, and $p(x, x)=0$ for all $x \in X$. Then the following statements are equivalent:

(i) $G$ is weakly connected;

(ii) Each orbitally $p$-continuous $(p, \varphi)-\widetilde{G}$-contraction is a $p$-Picard operator;

(iii) Each orbitally $p$-continuous $(p, \varphi)-\widetilde{G}$-contraction has at most one fixed point in $X$. In particular, if $\widetilde{G}$ is disconnected, then there exists an orbitally $p$-continuous $(p, \varphi)$ $\widetilde{G}$-contraction that has at least two fixed points in $X$.

Proof (i) $\Rightarrow$ (ii): It follows from Theorem 3 .

(ii) $\Rightarrow$ (iii): It is trivial.

(iii) $\Rightarrow$ (i): According to the proof of Theorem 1, it suffices to show that the self-map $f$ is orbitally $p$-continuous. To this end, let $x, y \in X$ and $\left\{a_{n}\right\}$ be a sequence of positive integers such that $f^{a_{n}} x \stackrel{p}{\rightarrow} y$. Then $\left\{f^{a_{n}} x\right\}$ is either the constant sequence $x_{0}$ or the constant sequence $y_{0}$. If the former holds, then $p\left(x_{0}, y\right)=0$. Since $p\left(x_{0}, x_{0}\right)=0$, it follows by Lemma 1 that $y=x_{0}$. Therefore,

$$
f\left(f^{a_{n}} x\right)=x_{0} \stackrel{p}{\rightarrow} x_{0}=f y .
$$

Otherwise, if the latter holds, a similar argument shows that $f$ is orbitally $p$-continuous.

Theorem 4 Let $X$ be p-complete and separated, and $f: X \rightarrow X$ be a graph orbitally $p$-continuous $(p, \varphi)$ - $\widetilde{G}$-contraction. Then

(i) for each $x \in X_{f}$, there exists an $x^{*} \in \operatorname{Fix}(f)$ such that $f^{n} y \stackrel{p}{\rightarrow} x^{*}$ for all $y \in[x]_{\widetilde{G}}$.

(ii) $\operatorname{Fix}(f) \neq \emptyset$ if and only if $X_{f} \neq \emptyset$.

(iii) If $X_{f} \neq \emptyset$ and $G$ is weakly connected, $f$ is a $p$-Picard operator. 
Proof (i) Let $x \in X$ be such that $(x, f x) \in E(\widetilde{G})$, and let $y \in[x]_{\widetilde{G}}$. Since $X$ is $p$-complete, by Proposition 1, $\left\{f^{n} x\right\}$ and $\left\{f^{n} y\right\}$ are $p$-convergent to a same point $x^{*} \in X$. Moreover, $\left(f^{n} x, f^{n+1} x\right) \in E(\widetilde{G})$ for all $n \geq 1$. Since $f$ is graph orbitally $p$-continuous, it follows that $f\left(f^{n} x\right) \stackrel{p}{\rightarrow} f x^{*}$. On the other hand, because

$$
f\left(f^{n} x\right)=f^{n+1} x \stackrel{p}{\rightarrow} x^{*}
$$

Lemma 1 ensures that $x^{*}=f x^{*}$, that is, $x^{*} \in \operatorname{Fix}(f)$.

(ii) If $X_{f} \neq \emptyset$, then, by (i), $\operatorname{Fix}(f) \neq \emptyset$. Conversely, suppose that $\operatorname{Fix}(f) \neq \emptyset$. Since $\Delta(X) \subseteq$ $E(\widetilde{G})$, it follows that $X_{f} \neq \emptyset$.

(iii) If $x \in X_{f}$, since $G$ is weakly connected, we have $[x]_{\widetilde{G}}=X$. Hence, by (i), there exists an $x^{*} \in \operatorname{Fix}(f)$ such that $f^{n} y \stackrel{p}{\rightarrow} x^{*}$ for all $y \in[x]_{\widetilde{G}}=X$. Now, similar to the proof of Theorem 2, it is seen that $x^{*}$ is the only fixed point for $f$.

Remark 4 In all theorems and corollaries above, setting $G=G_{0}\left(G=G_{1}\right)$, we get the usual (ordered) version of fixed point theorems in (partially ordered) uniform spaces.

\section{Competing interests}

The authors declare that they have no competing interests.

\section{Authors' contributions}

All authors read and approved the final manuscript.

Received: 25 April 2012 Accepted: 28 September 2012 Published: 17 October 2012

\section{References}

1. Aamri, M, El Moutawakil, D: Common fixed point theorems for $E$-contractive or E-expansive maps in uniform spaces. Acta Math. Acad. Paedagog. Nyházi. (N.S.) 20,83-91 (2004)

2. Jachymski, J: The contraction principle for mappings on a metric space with graph. Proc. Am. Math. Soc. 136, 1359-1373 (2008)

3. Bojor, F: Fixed point of $\varphi$-contraction in metric spaces endowed with a graph. An. Univ. Craiova, Ser. Mat. Inform. 37, 85-92 (2010)

4. Nicolae, A, O'Regan, D, Petruşel, A: Fixed point theorems for singlevalued and multivalued generalized contractions in metric spaces endowed with a graph. Georgian Math. J. 18, 307-327 (2011)

5. Altun, I, Imdad, M: Some fixed point theorems on ordered uniform spaces. Filomat 23, 15-22 (2009)

6. Tarafdar, E: An approach to fixed-point theorems on uniform spaces. Trans. Am. Math. Soc. 191, $209-225$ (1974)

7. Turkoglu, D, Binbasioglu, D: Some fixed-point theorems for multivalued monotone mappings in ordered uniform spaces. Fixed Point Theory Appl. 2011, Article ID 186237 (2001)

8. Willard, S: General Topology. Addison-Wesley, London (1970)

9. Bondy, JA, Murthy, USR: Graph Theory. Springer, New York (2008)

10. Petrusel, A, Rus, IA: Fixed point theorems in ordered L-spaces. Proc. Am. Math. Soc. 134, 411-418 (2006)

11. Gwóźdź-Łukawska, G, Jachymski, J: IFS on a metric space with a graph structure and extensions of the Kelisky-Rivlin theorem. J. Math. Anal. Appl. 356, 453-463 (2009) 